\title{
Wieviel MRT braucht der Neuropädiater?
}

Das MRT hat einen hohen Stellenwert in der Neuropädiatrie, und eine erhebliche Zahl neuropädiatrischer Patienten erhält insbesondere kranielle MRT-Untersuchungen (cMRT). Häufige Indikationen sind Kopfschmerzen, Krampfanfälle und Entwicklungsstörungen. Der Vortrag widmet sich der Frage nach der Indikationsstellung bei den genannten Symptomen.

Präsentiert sich ein Kind mit Kopfschmerzen, so muss entschieden werden, ob eine Bildgebung, die dann in der Regel mit cMRT erfolgt, durchgeführt werden soll und wie dringlich die Untersuchung ist. Häufig handelt es sich um primäre Kopfschmerzen oder gutartige sekundäre Kopfschmerzen, beispielsweise im Rahmen eines Luftweginfekts. Wichtig ist es, sekundäre Kopfschmerzen mit nicht benigner Ursache zu identifizieren. Dazu gehören Hirntumoren, Meningitis, Pseudotumor cerebri, Infarkt, subdurales Empyem, Hirnabszess und Shunt-Dysfunktion (Calder K, Kokorowski P, Tran et al. Pediatr Emerg Care 2003; 19: 320 - 328; Conicella E, Raucci U, Vanacore $\mathrm{N}$ et al. Headache 2008; 48: $1005-1011$; Dunn B, McCalla C, Hiestand B et al. Pediatr Emerg Care 2013; 29: 1283 - 1286; Trofi- mova A, Vey BL, Mullins ME et al. Am J Roentgeno 2018; 210: 8 - 17). In einer amerikanischen Arbeit (Trofimova A, Vey BL, Mullins ME et al. Am J Roentgeno 2018; 210: 8 - 17) wird für die Indikationsstellung zur cMRT-Untersuchung folgender Algorithmus vorgeschlagen: Eigener Algorithmus für Traumata; Infektionsursachensuche bei Fieber, bei V.a. Meningitis Lumbalpunktion, falls keine Kontraindikationen bestehen; cMRT bei nichttraumatischen Kopfschmerzen ohne Fieber nur, wenn Warnzeichen identifiziert werden. Zu den Warnzeichen können beispielsweise (Nüchtern-) Erbrechen oder fokale neurologische Symptome gehören.

Betrachtet man die Zahl pathologischer Befunde in der pädiatrischen Population, so zeigten sich beispielsweise in einer Studie, die 478 Patienten mit überwiegend Migräne und Spannungskopfschmerzen einschloss, bei 31,4\% auffällige Befunde, wobei es sich überwiegend um Zufallsbefunde handelte. 5 Patienten hatten Hirntumoren (Gurkas E, Karalok ZS, Taskın BD et al. Arch Argent Pediatr 2017; 115: e349-e355). Bei primären Kopfschmerzen erfolgt eine cMRT-Untersuchung rela- tiv häufig auf Wunsch von Eltern und/oder Patient. Hier sollte im Vorfeld die Möglichkeit von unklaren Zufallsbefunden und der Umgang damit besprochen werden (Roser T, Bonfert M, Ebinger F et al. Neuropediatrics 2013; 44: 34 - 39).

Bei Krampfanfällen muss ebenfalls über die Indikation für eine Bildgebung und über die Dringlichkeit entschieden werden. Diese ergibt sich wegen der Möglichkeit akut behandlungsbedürftiger Befunde vor allem bei einem ersten fokalen Krampfanfall. In einer retrospektiven Analyse aus Katar von 69 Kindern, die nach einem ersten afebrilen Krampfanfall eine Bildgebung erhielten, zeigte sich bei $33 \%$ eine Pathologie. Das Risiko für einen pathologischen Befund war bei einem Alter < 2 Jahren und prolongiertem Krampfanfall signifikant erhöht (Al-Shami R, Khair AM, Elseid M et al. Seizure 2016; 43: $26-31$ ). In einer US-amerikanischen Studie, die 177 Kinder mit erstem Krampfanfall, der sich als Status epilepticus manifestierte, einschloss, zeigte sich in der Bildgebung bei $36 \%$ eine Pathologie. Eine Pathologie, aus der sich ein akuter Behandlungsbedarf ableitete, hatten in der Studie 8,5\% der Kinder, u. a. Blutung, 
Ödem, ADEM, Infarkt und Abszess (Lyons TW, Johnson KB, Michelson KA et al. Seizure 2016; 35: 4-10). Die Studie identifizierte ein höheres Alter und eine längere Krampfdauer als Risikofaktoren für eine akut behandlungsbedürftige Pathologie.

Eine deutsche Studie identifizierte bei 248 Kindern in $23 \%$ eine wahrscheinlich epileptogene Läsion in der cMRT-Untersuchung (Tews W, Weise S, Syrbe $S$ et al. Klin Padiatr; 227: 84 -88). Bei Patienten mit initial unauffälliger cMRT-Untersuchung und fehlender Anfallsfreiheit unter Medikation sollte eine hochauflösende cMRTUntersuchung erfolgen, um die Frage nach möglichen epilepsiechirurgischen Optionen klären zu können. Beispielsweise hatten 29/44 (66\%) epilepsiechirurgischer Patienten mit initial unauffälliger cMRTUntersuchung eine Läsion in einer hochauflösenden CMRT-Untersuchung (Ahmed R, Rubinger L, Go C, Drake JM et al. Epilepsy Res 2018; 143: 113 -119).

Eine entscheidende Frage bei der Abklärung einer Entwicklungsstörung ist, inwieweit cMRT-Untersuchungen zu einer ätiologischen Zuordnung beitragen können. Eine relativ aktuelle Metaanalyse betrachtet die Ergebnisse aus 29 Studien, die Patienten im Alter von 0-18 Jahren mit globaler Entwicklungsverzögerung bzw. geistiger Behinderung einschlossen (Murias K, Moir A, Myers KA et al. Brain Dev 2017; 39: 644 -655). Bei insgesamt 2299 cMRT-Untersuchungen fanden sich in 38\% Auffälligkeiten. In 7,9\% der Fälle stellten sich Auffälligkeiten dar, die zur Klärung der Ursache der Entwicklungsstörung führten. In den unterschiedlichen Studien lag die Rate an Auffälligkeiten zwischen 0 und $98 \%$. Die Chance, dass sich bei einer cMRT-Untersuchung im Rahmen der Abklärung einer Entwicklungsstörung pathologische Befunde ergeben, ist deutlich erhöht, wenn zur Entwicklungsstörung andere neurologische Symptome hinzukommen. In einer 2011 veröffentlichten britischen Studie, die 157 Patienten einschloss, beispielsweise zeigten sich bei isolierter Entwicklungsstörung in 7,5\% spezifische strukturelle Auffälligkeiten, kamen neurologische Symptome hinzu, stieg das Risiko auf 28 \% (Griffiths PD, Batty R, Warren D et al. Eur Radiol 2011; 21: 1820 - 1830).

Zusammenfassend stellt die Untersuchung mittels cMRT ein wichtiges diagnostisches Werkzeug in der Neuropädiatrie dar. Die Kenntnis der Studienlage kann helfen, zu entscheiden, ob und mit welcher Dringlichkeit bei Symptomen wie Kopfschmerzen, Krampfanfällen und Ent- wicklungsstörungen eine cMRT-Untersuchung durchgeführt werden sollte.

Interessenkonflikt

Die Autoren geben an, dass kein Interessenkonflikt besteht.

Autorinnen/Autoren

Astrid Bertsche

Universitätsmedizin Rostock, Kinder- und

Jugendklinik, Neuropädiatrie

\section{Korrespondenzadresse}

Astrid Bertsche

Universitätsmedizin Rostock, Kinder- und

Jugendklinik, Neuropädiatrie

astrid.bertsche@med.uni-rostock.de

Bibliografie

DOI https://doi.org/10.1055/a-0943-1043 Online-Publikation: 2019

Fortschr Röntgenstr 2019; 191: S105-S106

(c) Georg Thieme Verlag KG, Stuttgart · New York ISSN 1433-5972 